\title{
Evolving Systems: An Outcome of Fondest Hopes and Wildest Dreams
}

\author{
Susan A. Frost ${ }^{1}$ \\ NASA Ames Research Center, Moffett Field, CA 94035 \\ and \\ Mark J. Balas ${ }^{2}$ \\ University of Wyoming, Laramie, WY 82071
}

\begin{abstract}
New theory is presented for evolving systems, which are autonomously controlled subsystems that self-assemble into a new evolved system with a higher purpose. Evolving systems of aerospace structures often require additional control when assembling to maintain stability during the entire evolution process. This is the concept of Adaptive Key Component Control that operates through one specific component to maintain stability during the evolution. In addition, this control must often overcome persistent disturbances that occur while the evolution is in progress. Theoretical results will be presented for Adaptive Key Component control for persistent disturbance rejection. An illustrative example will demonstrate the Adaptive Key Component controller on a system composed of rigid body and flexible body modes.
\end{abstract}

\section{Introduction}

$\mathrm{E}$ VOLVING systems are autonomously controlled subsystems which self-assemble into a new evolved system with a higher purpose $\mathrm{s}^{1-2}$. Evolving systems of aerospace structures often require additional control when assembling to maintain stability during the entire evolution process ${ }^{3-5}$. An adaptive key component controller has been shown to restore stability in evolving systems that would otherwise lose stability during evolution ${ }^{6-8}$. The adaptive key component controller uses a direct adaptation control law to restore stability to the evolving system through a subset of the input and output ports on one key component of the evolving system. The reader is directed to refs. 7-8 for a more detailed description of evolving systems. In this paper, we address the situation where persistent disturbances can appear in some components and must be mitigated by the adaptive key component controller. Such disturbances will often be attendant in actively controlled rendezvous and docking or component capture applications.

The control laws used by the adaptive key component controller to restore stability in an evolving system are guaranteed to have bounded gains and asymptotic tracking if the evolved system is almost strictly dissipative. Hence, it is desirable to know when the dissipativity traits of the subsystem components, including the key component, are inherited in an evolving system. We present results describing when an evolving system will inherit the almost strict passivity traits of its subsystem components. Then we will present an adaptive key component controller that restores asymptotic stability with bounded adaptive gains and mitigates the effect of persistent disturbances during evolution. The paper is organized as follows: the next section describes the framework for evolving systems, then the conditions under which dissipativity traits are inherited in evolving systems will be presented. Next, the design of adaptive key component disturbance accommodating control will be described. Finally, an illustrative example is presented to demonstrate the concepts presented earlier.

\footnotetext{
${ }^{1}$ Research Engineer, Intelligent Systems Division, POB 1, M/S 269-3, AIAA Member.

2 Department Head and Professor, Electrical and Computer Engineering Department, 1000 E. University Ave., AIAA Life Fellow.
} 


\section{Evolving Systems Framework}

\section{A. Mathematical Formulation of Evolving Systems}

A mathematical formulation of a nonlinear time-invariant evolving system is given here. Consider a system of $L$ components of individually, actively controlled subsystems which can be described by the following equations for the ith component:

$$
\left\{\begin{array}{l}
\dot{x}_{i}=f_{i}\left(x_{i}, u_{i}\right) \\
y_{i}=g_{i}\left(x_{i}, u_{i}\right)
\end{array}\right.
$$

where $i=1,2, \ldots, L$. The $i^{\text {th }}$ component has a Performance Cost Function $J_{i}$ and a Lyapunov Function $V_{i}$. These are the building blocks of the evolving System. When these individual components are joined to form an evolved system, the new entity becomes:

$$
\left\{\begin{array}{l}
\dot{x}=f(x, u) \\
y=g(x, u)
\end{array}\right.
$$

with $x=\left[x_{1} \ldots x_{L}\right]^{T}, y=\left[y_{1} \ldots y_{L}\right]^{T}$, Performance Cost Function $J$, and Lyapunov Function $V$. The $i^{\text {th }}$ component in the above evolved system is given by:

$$
\dot{x}_{i}=f_{i}\left(x_{i}, u_{i}\right)+\sum_{j=1}^{L} \varepsilon_{i j} f_{i j}\left(x_{i}, x_{j}, u_{j}\right)
$$

where $0 \leq \varepsilon_{i j} \leq 1$ and $f_{i j}\left(x_{i}, x_{j}, u_{j}\right)$ represents the interconnections between the $i^{\text {th }}$ and $j^{\text {th }}$ components. Note that when $\varepsilon_{i j}=0$, the system is in component form and when $\varepsilon_{i j}=1$, the system is fully evolved. As the system evolves, or joins together, the $\varepsilon_{i j}$ 's evolve from 0 to 1 .

The components of the evolving system are actively controlled by means of local control. Local control means dependence only on local state or local output information, i.e., $u_{i}=h_{i}\left(x_{i}\right)$ or $u_{i}=h_{i}\left(y_{i}\right)$. In general, the local controller on the $i^{\text {th }}$ component would have the form:

$$
\left\{\begin{array}{l}
u_{i}=h_{i}\left(y_{i}, z_{i}\right) \\
\dot{z}_{i}=l_{i}\left(y_{i}, z_{i}\right)
\end{array}\right.
$$

where $z_{i}$ is the dynamical part of the control law. Local control will be used to keep the components stable and to meet the individual component performance requirements, $J_{i}$.

Once the system is fully evolved, the $i^{\text {th }}$ component in the fully evolved system becomes:

$$
\dot{x}_{i}=f_{i}\left(x_{i}, u_{i}\right)+\sum_{j=1}^{L} f_{i j}\left(x_{i}, x_{j}, u_{j}\right)
$$

A state space version of the $i^{\text {th }}$ individual component of an evolving system where the components are connected through the states can be represented as: 


$$
\left\{\begin{array}{l}
\dot{x}_{i}=A_{i}\left(x_{i}\right)+B_{i}\left(x_{i}\right) u_{i}+\sum_{j=1}^{L} \varepsilon_{i j} A_{i j}\left(x_{i}, x_{j}\right) ; x_{i}(0) \equiv x_{0_{i}} \\
y_{i}=C_{i}\left(x_{i}\right)
\end{array}\right.
$$

where $i=1,2, \ldots L, x_{i} \equiv\left[x_{1}^{i} \ldots x_{n_{i}}^{i}\right]^{\mathrm{T}}$ is the component state vector, $u_{i} \equiv\left[u_{1}^{i} \ldots u_{m_{i}}^{i}\right]^{\mathrm{T}}$ is the control input vector, $y_{i} \equiv\left[y_{1}^{i} \ldots y_{p_{i}}^{i}\right]^{\mathrm{T}}$ is the sensor output vector, $\left(A_{i}\left(x_{i}\right), B_{i}\left(x_{i}\right), C_{i}\left(x_{i}\right)\right)$ are vector fields of dimension $n_{i} \times n_{i}, n_{i} \times m_{i}$, and $p_{i} \times n_{i}$, respectively, and the connection forces between components are represented in the $n_{i} \mathrm{x} n_{j}$ connection matrix, $A_{i j}\left(x_{i}, x_{j}\right)$ with $\varepsilon_{j i}=\varepsilon_{i j}$. The state space representation of the evolved system then becomes:

$$
\left\{\begin{array}{l}
\dot{x}=A(x)+B(x) u \\
y=C(x)
\end{array}\right.
$$

which will also be written as $(A(x), B(x), C(x))$.

\section{B. Characteristics and control of evolving systems}

We say a subsystem trait, such as stability, is inherited when the evolved system retains the characteristic of the trait from the subsystem. Previous papers have examined the inheritance of stability and shown that stability is not a generally inherited trait in evolving systems ${ }^{3-5}$. Control can be used in evolving systems to maintain stability in a system that would fail to inherit stability during evolution.

Key component control has been proposed to restore stability in evolving systems that would otherwise lose stability during evolution. The approach used for key component control design is for the control and sensing of the components to remain local and unaltered except in the case of one key component which has additional local

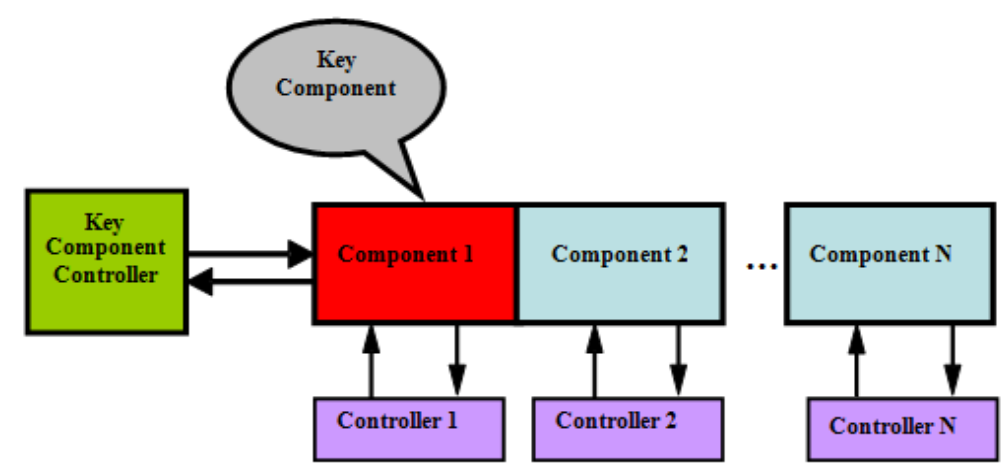

Fig. 1. Evolving system with key component controller. control added to stabilize the system during evolution. The key component controller operates solely through a single set of input-output ports on the key component, see Figure 1.

Only the key component of the evolving system needs modification to restore the inheritance of stability. A clear advantage of the key component design is that components can be reused in many different configurations of evolving systems without the need for component redesign. The reuse of components which are space-qualified, or at least previously designed and unit tested, could reduce the overall system development and testing time and should result in a higher quality system with potentially significant cost savings and risk mitigation.

In many aerospace environments and applications, the parameters of a system are poorly known and difficult to obtain. Adaptive key component controllers, which make use of a direct adaptation control law, are a good design choice for restoring stability in evolving systems where access to precisely known parametric values is limited. The sufficient condition for a nonlinear evolving system with an adaptive key component controller to be guaranteed to have bounded gains and to have asymptotic output tracking is that the system be almost strictly dissipative. So, we are interested in the conditions under which the inheritance of almost strict dissipativity can be guaranteed in evolving systems. The next section gives definitions and theorems related to dissipativity. 


\section{Inheritance of Almost Strict Dissipativity in Evolving Systems}

Inheritance of almost strict dissipativity of subsystems is desirable in evolving systems that use an adaptive key component controller to restore stability. Consider a nonlinear system given by Eq. 7. We say this system is Strictly Dissipative when there exists $V(x)>0 \forall x \neq 0$ such that the Lie derivatives satisfy:

$$
\left\{\begin{array}{l}
L_{A} V \equiv \nabla V A(x) \leq-S(x) \forall x \\
L_{B} V \equiv \nabla V B(x)=C^{T}(x)
\end{array}\right.
$$

where $\nabla V \equiv$ gradient $V$.

The function $V(x(t))$ is called the Storage Function for Eq. 7, and the above says that the storage rate is always less than the external power. This can be seen by taking the time derivative of $V(x(t))$ to obtain:

$$
\begin{aligned}
\dot{V} & \equiv \nabla V[A(x)+B(x) u] \\
& \leq-S(x)+C^{T}(x) u \\
& =-S(x)+\langle y, u\rangle
\end{aligned}
$$

Taking $u \equiv 0$, it is easy to see that Eq. 9 implies the first equation in Eq. 8 but not necessarily the second equation; so Eq. 8 implies Eq. 9 but not conversely. They are only equivalent if the first equation in Eq. 8 is an equality. (When equality holds in Eqs. 8 and 9, the property is known as Strict Passivity.)

We will say a system $(u, y)$ is Almost Strictly Dissipative $(A S D)$ when there is some output feedback, $u=G_{*} y+u_{r}$, that results in the following being strictly dissipative:

$$
\left\{\begin{array}{l}
\dot{x}=A_{C}(x)+B(x) u_{r} \\
A_{C}(x) \equiv A(x)+B(x) G_{*} C(x) \\
y=C(x)
\end{array}\right.
$$

Now if each component is ASD, then we have

$$
\left\{\begin{array}{l}
\nabla V_{i}\left[A_{i}\left(x_{i}\right)+B_{i}\left(x_{i}\right) G_{i} C_{i}\left(x_{i}\right)\right] \leq-S_{i}\left(x_{i}\right)+\sum_{j=1}^{L} \varepsilon_{i j} \nabla V_{i} A_{i j}\left(x_{j}, u_{j}\right) \\
\nabla V_{i} B_{i}\left(x_{i}\right)=C_{i}^{T}\left(x_{i}\right)
\end{array}\right.
$$

where $\nabla V_{i} \equiv$ gradient $\left(V_{i}\right)$. Due to the interconnection terms, Eq. 11 is not necessarily Strictly Dissipative. However, in some circumstances, the interconnection terms have a special form and ASD is inherited when the system evolves.

Suppose we have a pair of subsystems of the form:

$$
\left\{\begin{array}{l}
\dot{x}_{i}=A_{i}\left(x_{i}\right)+\varepsilon B_{i}\left(x_{i}\right) u_{i}+B_{i}^{A}\left(x_{i}\right) u_{i}^{A} \\
y_{i}=C_{i}\left(x_{i}\right) \\
y_{i}^{A}=C_{i}^{A}\left(x_{i}\right)
\end{array}\right.
$$


where $i=1,2$ and both subsystems $\left(\left[\begin{array}{l}u_{1} \\ u_{1}^{A}\end{array}\right],\left[\begin{array}{l}y_{1} \\ y_{1}^{A}\end{array}\right]\right)$ and $\left(\left[\begin{array}{l}u_{2} \\ u_{2}^{A}\end{array}\right],\left[\begin{array}{c}y_{2} \\ y_{2}^{A}\end{array}\right]\right)$ have storage functions $V_{i}$. We have the following result:

Theorem 1: If the subsystems $\left(u_{1}^{A}, y_{1}^{A}\right)$ and $\left(u_{2}^{A}, y_{2}^{A}\right)$ are ASD and

$$
\nabla V_{i} B_{i}\left(x_{i}\right)=C_{i}^{T}\left(x_{i}\right) ; i=1,2
$$

then the resulting feedback connection, $y_{1}=u_{2}$ and $\mathrm{u}_{1}=-y_{2}$, will leave the composite system $\left(u_{A} \equiv\left[\begin{array}{l}u_{1}^{A} \\ u_{2}^{A}\end{array}\right], y_{A} \equiv\left[\begin{array}{l}y_{1}^{A} \\ y_{2}^{A}\end{array}\right]\right)$ almost strictly passive.

Proof: See appendix.

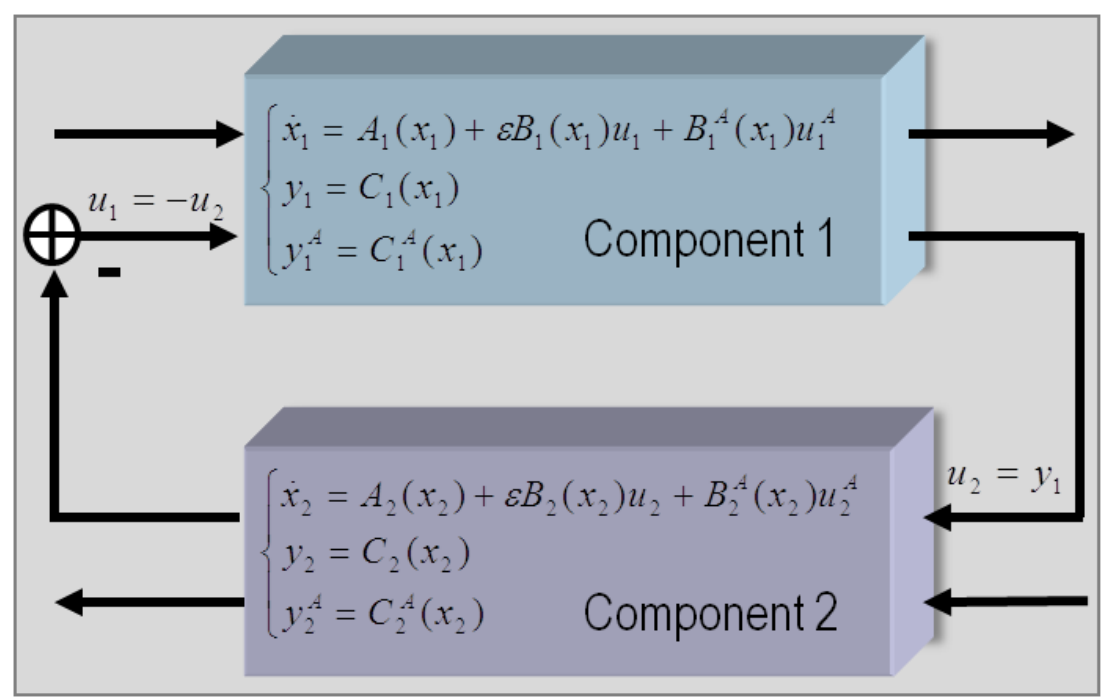

The physical connection of components in evolving systems is equivalent to the feedback connection of the admittance of one component to the impedance of the other component ${ }^{3,5}$. See fig. 2 for a schematic of the admittance-impedance connection between two evolving system components. The $A$ superscript on the input and output variables is used to indicate that the component is in admittance-impedance form. For a nonlinear evolving system with components $\left(u_{1}, y_{1}\right)$ and $\left(u_{2}, y_{2}\right)$ connected in admittance-impedance form, Theorem 1 shows that ASD is an inherited property.

Fig. 2. Admittance-Impedance feedback connection of evolving systems.

\section{Adaptive Key Component Control with Persistent Disturbance Mitigation}

This section describes the adaptive key component control with persistent disturbance mitigation. The key component is chosen to be component \#1 and will be modeled by the following nonlinear evolving system component with an external persistent disturbance:

$$
\left\{\begin{array}{l}
\dot{x}_{1}=A_{1}\left(x_{1}\right)+\varepsilon B_{1}\left(x_{1}\right) u_{1}+B_{1}^{A}\left(x_{1}\right) u_{1}^{A}+\Gamma_{1}\left(x_{1}\right) u_{D} \\
y_{1}=C_{1}\left(x_{1}\right) \\
y_{1}^{A}=C_{1}^{A}\left(x_{1}\right)
\end{array}\right.
$$

which will be respresented by the triple $\left(A_{1}\left(x_{1}\right), B_{1}\left(x_{1}\right), B_{1}^{A}\left(x_{1}\right), \Gamma_{1}\left(x_{1}\right), C_{1}^{A}\left(x_{1}\right)\right)$. All vector fields in Eq. 14 will have the appropriate compatible dimensions and be smooth in their arguments with a single equilibrium point at 0 in 
a neighborhood $\mathrm{U}$. The persistent disturbance input vector $u_{D}(t)$ is $\mathrm{N}_{\mathrm{D}}$-dimensional and will be thought to come from the following Disturbance Generator:

$$
\left\{\begin{array}{l}
u_{D}=\Theta z_{D} \\
\dot{z}_{D}=F z_{D} ; z_{D}(0)=z_{0}
\end{array}\right.
$$

where the disturbance state $z_{D}(t)$ is $\mathrm{N}_{\mathrm{D}}$-dimensional. Such descriptions of persistent disturbances were first used to describe signals of known form but unknown amplitude ${ }^{10}$. For example, step disturbances yield $\Theta=1$ and $F=0$ while sinusoidal disturbances can be described by $\Theta=\left[\begin{array}{ll}1 & 0\end{array}\right]$ and $F=\left[\begin{array}{cc}0 & 1 \\ -\omega_{D}^{2} & 0\end{array}\right]$ where the frequency $\omega_{D}$ is known but the amplitudes are not known.

Assume that the disturbance generator parameter $F$ is known. In many cases this is not a severe restriction, e.g. step disturbances and sinusoidal disturbances are common forms of persistent disturbances. It turns out that it is better to rewrite the above in the following equivalent form:

$$
\left\{\begin{array}{l}
u_{D}=\Theta z_{D} \\
z_{D}=L \varphi_{D}
\end{array}\right.
$$

where $\varphi_{D}$ is a vector composed of the known basis functions for the solutions of Eq. 15 and $(L, \Theta)$ need not be known. The following example illustrates the basis function form for the disturbance generator:

$$
\begin{aligned}
z_{D}(t) & =e^{F t} z_{D}(0) \\
& =\left[\varphi_{1}(t), \varphi_{2}(t), \ldots, \varphi_{N_{D}}(t)\right] z_{D}(0) \\
& =\sum_{i=1}^{N_{D}} z_{D}^{i} \varphi_{i}(t)=L \varphi_{D}
\end{aligned}
$$

Note that $L$ is directly related to $F$ via its columns but not to $\theta$. Some rearrangement of the entries in the columns of $F$ is needed to create $\varphi_{D}$. A simple example of the above is given by the following:

$$
\left\{\begin{aligned}
\dot{x} & =\left[\begin{array}{ll}
0 & 1 \\
0 & 0
\end{array}\right] x+\left[\begin{array}{l}
0 \\
1
\end{array}\right] u+\left[\begin{array}{l}
1 \\
1
\end{array}\right] u_{D} \\
u_{D} & =a \sin \left(\omega_{D} t+b\right) \\
& =a_{1} \sin \left(\omega_{D} t\right)+a_{2} \cos \left(\omega_{D} t\right)
\end{aligned}\right.
$$

Assume component \#1 $\left(\left[\begin{array}{c}u_{1} \\ u_{1}^{A}\end{array}\right],\left[\begin{array}{c}y_{1} \\ y_{1}^{A}\end{array}\right]\right)$ is ASD. Also let the following matching condition be true:

$$
R\left(\Gamma_{1}\left(x_{1}\right)\right) \subseteq R\left(B_{1}^{A}\left(x_{1}\right)\right)
$$


The matching condition means that there exists $H_{*}$ such that $B_{1}^{A}\left(x_{1}\right) H_{*}=\Gamma_{1}\left(x_{1}\right)$. Let component \#2 represent the remainder of the evolving system and assume it is strictly dissipative by choice of local controllers, that is:

$$
\left\{\begin{array}{l}
\dot{x}_{2}=A_{2}\left(x_{2}\right)+\varepsilon B_{2}\left(x_{2}\right) u_{2} \\
y_{2}=C_{2}\left(x_{2}\right)
\end{array}\right.
$$

The components are in admittance-impedance form so when they are joined $u_{1}=-y_{2}$ and $u_{2}=y_{1}$. The Adaptive Key Component Controller with Disturbance Mitigation works through the control input-output ports $\left(u_{1}^{A}, y_{1}^{A}\right)$ of component \#1 and is given by:

$$
\left\{\begin{array}{l}
u_{1}^{A}=G_{e} y_{1}^{A}+G_{D} \varphi_{D} \\
\dot{G}_{e}=-y_{1}^{A}\left(y_{1}^{A}\right)^{T} \gamma_{e} ; \gamma_{e}>0 \\
\dot{G}_{D}=-y_{1}^{A}\left(\varphi_{D}\right)^{T} \gamma_{D} ; \gamma_{D}>0
\end{array}\right.
$$

This produces $x \equiv\left[\begin{array}{l}x_{1} \\ x_{2}\end{array}\right] \underset{t \rightarrow \infty}{\longrightarrow} 0$ with bounded adaptive gains $\left(G_{e}, G_{D}\right)$ as the following convergence theorem shows:

Theorem 2: Assume that $V_{1}$ and $V_{2}$ are positive $\forall x \neq 0$ and radially unbounded, and $(A(x), B(x), C(x))$ are continuous functions of $x$ and $S(x)$, above, is positive $\forall x \neq 0$ and has continuous partial derivatives in $x$. Furthermore, assume:

1) The conditions of Theo.1 are satisfied; so that $\left(\left[\begin{array}{c}u_{1} \\ u_{1}^{A}\end{array}\right],\left[\begin{array}{c}y_{1} \\ y_{1}^{A}\end{array}\right]\right)$ is Almost Strictly Dissipative (ASD)

2) The following matching condition is met: $R\left(\Gamma_{1}\left(x_{1}\right)\right) \subseteq R\left(B_{1}^{A}\left(x_{1}\right)\right)$

3) $\varphi_{D}$ is bounded (or equivalently, $\mathrm{F}$ has only simple imaginary poles and no right half-plane poles)

Then the adaptive Controller given by Eq. 20 produces $x \equiv\left[\begin{array}{l}x_{1} \\ x_{2}\end{array}\right] \underset{t \rightarrow \infty}{\longrightarrow} 0$ with bounded adaptive gains $\left(G_{e}, G_{D}\right)$ when Component 1 is joined with Component 2 into an evolved system and the outputs are $y_{i}=C_{i}\left(x_{i}\right) \underset{t \rightarrow \infty}{\longrightarrow} 0$.

Proof: See Appendix.

It should be noted that the above results might only hold on a neighborhood $N_{i}\left(0, r_{i}\right) \equiv\left\{x_{i} /\left\|x_{i}\right\|<r_{i}\right\}$. Then the stability in Theo. 2 is only locally asymptotic to the origin.

In the next section, an illustrative example will be given. 


\section{Illustrative Example}

Example 1 is a two component linear flexible structure evolving system, see fig. 3. The components of Example 1 are stable when they are unconnected components, but the evolving system fails to inherit the stability of the components. This example will be used to demonstrate the inheritance and lack of inheritance of almost strict

Component 1

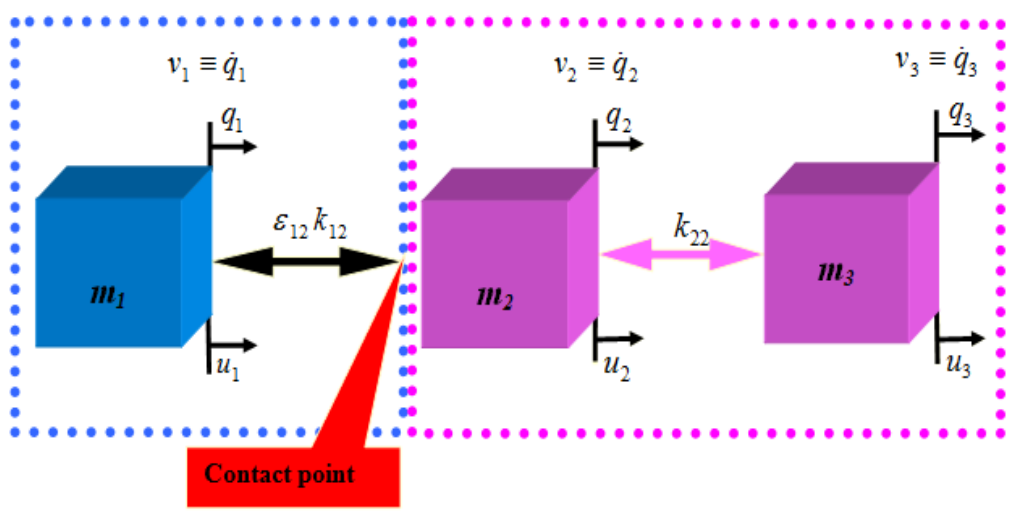

Fig. 3. A two component flexible structure Evolving System. dissipativity in evolving systems.

The dynamical equations for the components of Example 1 are:

$$
\begin{aligned}
& \left\{\begin{array}{l}
m_{1} \ddot{q}_{1}=u_{1}-\varepsilon_{12} k_{12}\left(q_{1}-q_{2}\right) \\
y_{1}=\left[q_{1}, \dot{q}_{1}\right]^{\mathrm{T}}
\end{array}\right. \\
& \left\{\begin{array}{l}
m_{2} \ddot{q}_{2}=u_{2}-\varepsilon_{12} k_{12}\left(q_{2}-q_{1}\right) \\
-k_{22}\left(q_{2}-q_{3}\right) \\
m_{3} \ddot{q}_{3}=u_{3}-k_{22}\left(q_{3}-q_{2}\right) \\
y_{2}=\left[q_{2}, \dot{q}_{2}\right]^{\mathrm{T}} \\
y_{3}=\left[q_{3}, \dot{q}_{3}\right]^{\mathrm{T}}
\end{array}\right.
\end{aligned}
$$

with $m_{1}=30, m_{2}=1, m_{3}=1, k_{12}=4$, and $k_{22}=1$. Example 1 has the following controllers:

$$
\left\{\begin{array}{l}
u_{1}=-(0.9 s+0.1) q_{1} \\
u_{2}=-\left(\frac{0.1}{s}+0.2 s+0.5\right) q_{2} \\
u_{3}=-(0.6 s+1) q_{3}
\end{array}\right.
$$

When two components join to form an Evolved System, at their point of contact, their velocities are equal and the forces exerted are equal and opposite. If the two components are given by $\left(f_{1}, v_{1}\right)$ and $\left(f_{2}, v_{2}\right)$, then the contact dynamics of the Evolved System can be represented by:

$$
\left\{\begin{array}{l}
f_{1}=-f_{2} \\
v_{1} \equiv \dot{q}_{1}=v_{2} \equiv \dot{q}_{2}
\end{array}\right.
$$

This connection is modeled as the admittance of one component connected in feedback with the impedance of the other component, as described in section II. When we use this idea of the joining of two components of an Evolving System as the feedback connection of their admittance and impedance, we can apply Theorem 1 from above to determine whether almost strict dissipativity is inherited by the Evolved System. The subsystem components from Example 1 are stable in closed-loop form when they are unconnected, i.e., $\varepsilon_{12}=0$. When $\varepsilon_{12}=1$, the system is fully evolved and it has a closed-loop eigenvalue at 0.17 , resulting in an unstable Evolved System. 


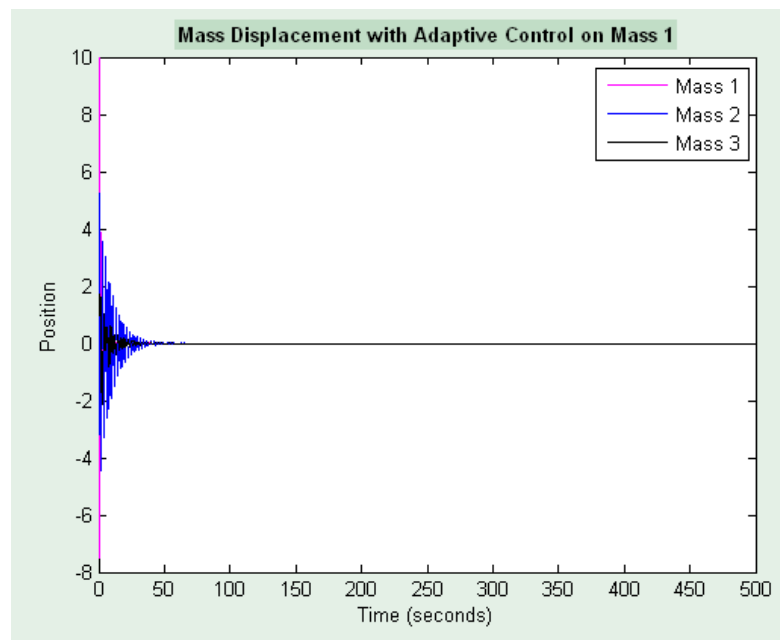

A Simulink model was created to implement an adaptive key component controller for Example 1 as described in the previous section. Simulations were run in which the connection parameter, $\varepsilon_{12}$, ranged from 0 to 1 , allowing the system to go from unconnected components to a fully Evolved System. The key component controller was able to maintain system stability during the entire evolution process when it used the input-output ports on mass 1 of component 1 , see fig. 4 . When component 1 was the key component, $(\bar{A}, \bar{B}, \bar{C})$ is ASPR.

When the key component controller was located on component 2 and used the input-output ports on mass 3, stability was not maintained, see Figure 5. The adaptive key component controller was not able to restore stability on mass 3 because that system was not ASPR, i.e., it had nonminimum phase zeros at $0.00515 \pm 0.2009 \mathrm{i}$.

A second study was performed where a proportional integral (PI) controller is used to control a rigid body. The plant has additional flexible body modes. The perturbation parameter $\varepsilon$ is used to connect or disconnect the flexible modes. In this way, a system with flexible modes that vary depending on the operating condition can be modeled. For the example described here, the system needs the adaptive key component control to maintain stability of the rigid and flex modes. Finally, one flex mode is defined that causes a non-minimum phase zero to appear in the plant when the feedback control is used. A residual mode filter is designed for this mode and successfully applied to remove the system that is causing the dissipativity condition to not be satisfied. The final paper will provide details of these models and results of the simulation.

\section{Conclusion}

We have presented a result (Theorem 1) describing when an evolving system will inherit the almost strict dissipativity traits of its subsystem components. An example was given of successful inheritance of almost strict dissipativity and failed inheritance of almost strict dissipativity. This result allows a control system designer to determine a sufficient condition for an evolving system to use an adaptive key component controller to restore stability. We also presented a convergence result (Theorem 2) for an adaptive key component controller to restore stability during evolution and mitigate persistent disturbances. An illustrative example of a system with a rigid mode and flexible modes is used to demonstrate these ideas.

\section{Appendix}

\section{Proof of Theorem 1:}

Let $\left(u_{i}^{A}, y_{i}^{A}\right)$ be ASD. From (9) and (11), there exists $G_{i}^{*}$ such that

$$
\begin{aligned}
\nabla V_{i} A_{i}^{C}\left(x_{i}\right) & \equiv \nabla V_{i}\left[A_{i}\left(x_{i}\right)+B_{i}^{A}\left(x_{i}\right) G_{i}^{*} C_{i}^{A}\left(x_{i}\right)\right] \\
& \leq-S_{i}\left(x_{i}\right)+\varepsilon_{i j} \nabla V_{i} A_{i j}\left(x_{i}, u_{i}, u_{i}^{A}\right) \\
\nabla V_{i} B_{i}^{A}\left(x_{i}\right) & =C_{i}^{A}\left(x_{i}\right)^{\mathrm{T}}
\end{aligned}
$$


If we connect $\left(u_{1}, y_{1}\right)$ in feedback with $\left(u_{2}, y_{2}\right)$, then $y_{1}=u_{2}$ and $\mathrm{u}_{1}=-y_{2}$ and, use (12) and (13), then we have $\quad \nabla V_{1} A_{12}\left(x_{1}, u_{1}, u_{1}^{A}\right)=\nabla V_{1} B_{1}\left(x_{1}\right) u_{1}=C_{1}^{T}\left(x_{1}\right)\left[-y_{2}\right]=-y_{1}^{T} y_{2} \quad$ and $\quad$ similarly, $\nabla V_{2} A_{21}\left(x_{2}, u_{2}, u_{2}^{A}\right)=y_{2}^{T} y_{1}$.

Let $x \equiv\left[\begin{array}{l}x_{1} \\ x_{2}\end{array}\right]$ and, from (12),

$$
\left\{\begin{aligned}
\dot{x} & =A(x)+B(x) u \\
& =\left[\begin{array}{l}
A_{1}^{C}\left(x_{1}\right)+\varepsilon_{12} A_{12}\left(x_{2}\right) \\
A_{2}^{C}\left(x_{2}\right)+\varepsilon_{21} A_{21}\left(x_{1}\right)
\end{array}\right] \\
& +\left[\begin{array}{cc}
B_{1}^{A}\left(x_{1}\right) & 0 \\
0 & B_{2}^{A}\left(x_{2}\right)
\end{array}\right]\left[\begin{array}{l}
u_{1}^{A} \\
u_{2}^{A}
\end{array}\right] \\
y & =\left[\begin{array}{l}
y_{1}^{A} \\
y_{2}^{A}
\end{array}\right]=C(x)=\left[\begin{array}{l}
C_{1}^{A}\left(x_{1}\right) \\
C_{2}^{A}\left(x_{2}\right)
\end{array}\right]
\end{aligned}\right.
$$

with $V=V_{1}+V_{2}$, using (13) and $\varepsilon_{j i}=\varepsilon_{i j} \equiv \varepsilon$ from (3),

$$
\begin{aligned}
\nabla V A(x) & =\left[\begin{array}{ll}
\nabla V_{1} & \nabla V_{2}
\end{array}\right]\left[\begin{array}{l}
A_{1}^{C}\left(x_{1}\right)+\varepsilon A_{12}\left(x_{2}\right) \\
A_{2}^{C}\left(x_{2}\right)+\varepsilon A_{21}\left(x_{1}\right)
\end{array}\right] \\
& =\nabla V_{1} A_{1}\left(x_{1}\right)+\varepsilon\left(-y_{1}^{T} y_{2}\right)+\nabla V_{2} A_{2}\left(x_{2}\right)+\varepsilon\left(y_{2}^{T} y_{1}\right) \\
& \leq-\left[S_{1}\left(x_{1}\right)+S_{2}\left(x_{2}\right)\right]+\varepsilon\left(-y_{1}^{T} y_{2}\right)+\varepsilon\left(y_{2}^{T} y_{1}\right) \\
& =-S(x)
\end{aligned}
$$

and

$$
\begin{aligned}
\nabla V B(x) & =\left[\nabla V_{1} \nabla V_{2}\right]\left[\begin{array}{cc}
B_{1}^{A}\left(x_{1}\right) & 0 \\
0 & B_{2}^{A}\left(x_{2}\right)
\end{array}\right] \\
& =\left[\begin{array}{l}
C_{1}^{A}\left(x_{1}\right) \\
C_{2}^{A}\left(x_{2}\right)
\end{array}\right]^{T}=C^{T}(x)
\end{aligned}
$$

Therefore $\left(u_{A} \equiv\left[\begin{array}{l}u_{1}^{A} \\ u_{2}^{A}\end{array}\right], y_{A} \equiv\left[\begin{array}{l}y_{1}^{A} \\ y_{2}^{A}\end{array}\right]\right)$ is ASD with output feedback $\left[\begin{array}{c}u_{1}^{A} \\ u_{2}^{A}\end{array}\right] \equiv\left[\begin{array}{cc}G_{1}^{*} & 0 \\ 0 & G_{2}^{*}\end{array}\right]\left[\begin{array}{l}y_{1}^{A} \\ y_{2}^{A}\end{array}\right]+\left[\begin{array}{l}u_{1}^{A r} \\ u_{2}^{A r}\end{array}\right]$ as desired. End of proof.

\section{Proof of Theorem 2:}

Since the physical connection of Component 1 to Component 2 is equivalent to the feedback connection $u_{1}=-y_{2}$ and $u_{2}=y_{2}$.

By Theo. 1 we have that the closed-loop system $\left(u_{1}^{A}, y_{1}^{A}\right)$ below is ASD: 


$$
\left\{\begin{array}{l}
\dot{x}_{1}=A_{1}\left(x_{1}\right)-\varepsilon B_{1}\left(x_{1}\right) C_{2}\left(x_{2}\right)+B_{1}^{A}\left(x_{1}\right) u_{1}^{A} \\
\dot{x}_{2}=A_{2}\left(x_{2}\right)+\varepsilon B_{2}\left(x_{2}\right) C_{1}\left(x_{1}\right) ; 0 \leq \varepsilon \leq 1 \\
y_{1}^{A}=C_{1}^{A}\left(x_{1}\right)
\end{array}\right.
$$

Rewrite (20), to obtain

$$
\left\{\begin{array}{l}
u_{1}^{A}=G_{e} y_{1}^{A}+G_{D} \varphi_{D}=G_{e}^{*} y_{1}^{A}+G_{D}^{*} \varphi_{D}+\Delta G \eta \\
\Delta G \equiv G-G^{*}=\left[\begin{array}{ll}
\Delta G_{e} & \Delta G_{D}
\end{array}\right] ; \eta \equiv\left[\begin{array}{l}
y_{1}^{A} \\
\varphi_{D}
\end{array}\right]^{w} \\
\Delta \dot{G}=\dot{G}=-y_{1}^{A}\left(y_{1}^{A}\right)^{T} \gamma ; \gamma \equiv\left[\begin{array}{cc}
\gamma_{e} & 0 \\
0 & \gamma_{D}
\end{array}\right]>0
\end{array}\right.
$$

Combining (21) and (22) yields:

$$
\left\{\begin{array}{l}
\dot{x}_{1}=A_{1}^{C}\left(x_{1}\right)-\varepsilon B_{1}\left(x_{1}\right) C_{2}\left(x_{2}\right) \\
+\left[B_{1}^{A}\left(x_{1}\right) G_{D}^{*}+\Gamma_{1}\left(x_{1}\right) \theta L\right] \varphi_{D}+B_{1}^{A}\left(x_{1}\right) w \\
\quad \text { with } w \equiv \Delta G \eta \\
\text { and } G_{D}^{*} \equiv-H_{*} \theta L \text { from }(19) \\
\quad \text { and } A_{1}^{C}\left(x_{1}\right) \equiv A_{i}\left(x_{i}\right)+B_{i}^{A}\left(x_{i}\right) G_{i}^{*} C_{i}^{A}\left(x_{i}\right) \\
\dot{x}_{2}=A_{2}\left(x_{2}\right)+\varepsilon B_{2}\left(x_{2}\right) C_{1}\left(x_{1}\right) ; 0 \leq \varepsilon \leq 1 \\
y_{1}^{A}=C_{1}^{A}\left(x_{1}\right)
\end{array}\right.
$$

Let $V=V_{1}+V_{2}$ and we have:

$$
\dot{V}=-S(x)+\left\langle y_{1}^{A}, w\right\rangle
$$

Form $V_{G} \equiv \frac{1}{2} \operatorname{tr}\left(\Delta G \gamma^{-1} \Delta G^{T}\right)$ and obtain from (22):

$$
\begin{aligned}
\dot{V}_{G} & \equiv \operatorname{tr}\left(\Delta \dot{G} \gamma^{-1} \Delta G^{T}\right) \\
& =-\operatorname{tr}\left(y_{1}^{A}\left(y_{1}^{A}\right)^{T} \Delta G^{T}\right) \\
& =-\operatorname{tr}\left(y_{1}^{A}(w)^{T}\right) \\
& =-\left\langle y_{1}^{A}, w\right\rangle
\end{aligned}
$$

Define: $V(x, \Delta G) \equiv V(x)+V_{G}(\Delta G)$ and, from (24) and (25), we have: 


$$
\begin{aligned}
\dot{V}(x, \Delta G) & \equiv \dot{V}(x)+\dot{V}_{G}(\Delta G) \\
& =-S(x)+\left\langle y_{1}^{A}, w\right\rangle-\left\langle y_{1}^{A}, w\right\rangle \\
& =-S(x) \leq 0
\end{aligned}
$$

This guarantees that all trajectories $(x, \Delta G)$ are bounded. If $\dot{V}(x, \Delta G)$ is uniformly continuous or $\ddot{V}(x, \Delta G)$ is bounded, then Barbalat's Lemma [10] yields:

$$
S(x) \underset{t \rightarrow \infty}{\longrightarrow} 0
$$

and the positivity and continuity of $S(x)$ imply that $x \equiv\left[\begin{array}{l}x_{1} \\ x_{2}\end{array}\right] \underset{t \rightarrow \infty}{\longrightarrow} 0$.

Consider

$$
\begin{aligned}
\ddot{V}(x, \Delta G) & =-\dot{S}(x) \\
& \leq|\dot{S}(x)| \\
& =\left|\frac{\partial S(x)}{\partial x} \dot{x}\right| \\
& \leq\left\|\frac{\partial S(x)}{\partial x}\right\|\|\dot{x}\| \\
& \leq\left\|\frac{\partial S(x)}{\partial x}\right\|\left[\|A(x)\|+\|B(x)\|\left\|w_{1}^{A}\right\|\right] \\
& \leq\left\|\frac{\partial S(x)}{\partial x}\right\|\left[\|A(x)\|+\|B(x)\|\|\Delta G\|\left\|C_{1}^{A}\left(x_{1}\right)\right\|\right]
\end{aligned}
$$

which is bounded because $(x, \Delta G)$ is bounded, $S(x)$ has continuous partial derivatives and $(A(x), B(x), C(x))$ are continuous, and a continuous function of bounded $x(t)$ is also bounded in $t$.

So, $y_{i}=C_{i}\left(x_{i}\right) \underset{t \rightarrow \infty}{\longrightarrow} 0$ because $C_{i}\left(x_{i}\right)$ is continuous. End of Proof.

\section{Acknowledgments}

The Subsonic Fixed Wing Project under NASA's Fundamental Aeronautics Program supported this work.

\section{References}

${ }^{1}$ M.J. Balas, S.A. Frost, and F.Y. Hadaegh, "Evolving Systems: A Theoretical Foundation," Proceedings AIAA Guidance, Navigation, and Control Conference, Keystone, CO, 2006.

${ }^{2}$ M. J. Balas and S. A. Frost, "An Introduction to Evolving Systems of Flexible Aerospace Structures," Proceedings IEEE Aerospace Conference, Big Sky, MT, 2007.

${ }^{3}$ S. A. Frost and M. J. Balas, "Stability Inheritance and Contact Dynamics of Flexible Structure Evolving Systems", Proceedings 17th IFAC Symposium on Automatic Control in Aerospace, Toulouse, France, 2007.

${ }^{4}$ M. J. Balas and S. A. Frost, "Evolving Systems: Inheriting Stability with Evolving Controllers", Proceedings $47^{\text {th }}$ Israel Annual Conference on Aerospace Sciences, Tel-Aviv, Israel, 2007.

${ }^{5}$ S.A. Frost and M.J. Balas, "Stabilizing Controllers for Evolving Systems with Application to Flexible Space Structures," Proceedings AIAA Guidance, Navigation, and Control Conference, Hilton Head, SC, 2007.

${ }^{6}$ S.A. Frost and M.J. Balas, "Adaptive Key Component Controllers for Evolving Systems," Proceedings AIAA Guidance, Navigation, and Control Conference, Honolulu, HI, 2008. 
${ }^{7}$ S.A. Frost and M.J. Balas, "Evolving Systems: Adaptive Key Component Control and Inheritance of Passivity and Dissipativity," Applied Mathematics and Computation, 217 (2010) 1034-1044.

${ }^{8}$ S.A. Frost and M.J. Balas, "Evolving Systems and Adaptive Key Component Control", chapter in Aerospace Technologies Advancements, Thawar T. Arif, ed., 2010, ISBN: 978-953-7619-96-1.

${ }^{9}$ R. J. Fuentes and Ml J. Balas, "Direct adaptive rejection of persistent disturbances," Journal of Mathematical Analysis and Applications, Vol. 251, No.1, 2000, pp. 28-39.

${ }^{10}$ CD Johnson, "Theory of Disturbance Accommodating Control," in Control \& Dynamic Systems, C.T. Leondes, editor, Vol. 12, 1976.

${ }^{11}$ V.M. Popov, Hyperstability of Control Systems, Springer, Berlin, 1978.

${ }^{12}$ M.J. Balas, S.A. Frost, and F.Y. Hadaegh, "Evolving Systems: A Theoretical Foundation," Proceedings ASME SMASIS, Scottsdale, AZ, Sept. 2011. 